\title{
Radical Copolymerization of Vinyl Ethers and Cyclic Ketene Acetals as a Versatile Platform to Design Functional Polyesters
}

\author{
Antoine Tardy, ${ }^{[a]}$ Jean-Claude Honoré, ${ }^{[a], \dagger}$ Johanna Tran, ${ }^{[b], \dagger}$ Didier Siri, ${ }^{[a]}$ Vianney Delplace ${ }^{[b]}$ Isabelle \\ Bataille, ${ }^{[c]}$ Didier Letourneur, ${ }^{[\mathrm{c}]}$ Josette Perrier, ${ }^{[\mathrm{d}]}$ Cendrine Nicoletti, ${ }^{[\mathrm{d}]}$ Marc Maresca,${ }^{[\mathrm{d}]}$ Catherine \\ Lefay, ${ }^{\left[{ }^{[a]}\right.}$ Didier Gigmes, ${ }^{[a]}$ Julien Nicolas, ${ }^{[b], *}$ Yohann Guillaneuf ${ }^{[a], *}$
}

\begin{abstract}
For the first time, the free-radical copolymerization of cyclic ketene acetals (CKAs) and vinyl ethers (VEs) was investigated as an efficient, yet simple approach for the preparation of functional aliphatic polyesters. The copolymerization of CKA and VE was first predicted to be quasi-ideal by DFT calculations. The theoretical prediction was experimentally confirmed by the copolymerization of 2-methylene-1,3-dioxepane (MDO) and butyl vinyl ether (BVE), leading to $r_{\mathrm{MDO}}=0.73$ and $r_{\mathrm{BVE}}=1.61$. We then illustrated the versatility of this approach by preparing different functional polyesters: (i) copolymers functionalized by fluorescent probes; (ii) amphiphilic copolymers grafted with poly(ethylene glycol) (PEG) side chains able to self-assemble into PEGylated nanoparticles; (iii) antibacterial films active against Gram+ and Gram- bacteria (including a multiresistant strain) and (iv) cross-linked bioelastomers with suitable properties for tissue engineering applications.
\end{abstract}

Aliphatic polyesters are commonly used as polymer-based biomaterials because of their well-established biocompatibility and biodegradability. ${ }^{[1-2]}$ Nevertheless such materials exhibited severe limitations because of their hydrophobic and/or semicrystalline properties and absence of functionality, that could otherwise be used for tuning their physical properties or for the coupling with various molecules of interest. The side-chain functionalization of aliphatic polyesters has thus been a topic of great interest over the past few years. but still requires many steps. ${ }^{[3-5]}$ For example, synthetic routes using functionalized lactones/carbonate prepared in many steps using bearing reactive groups-were copolymerized with traditional lactones/carbonates monomers, leading to a broad range of different biomaterials. ${ }^{[6-10]}$ Recently, elegant approaches were also developed to synthetize functionalized polyesters based on radical ring-opening copolymerization of cyclic ketene acetals (CKAs) with traditional vinyl monomers. ${ }^{[11-15]}$. CKA monomers

[a] Dr. A. Tardy, J.-C. Honoré, Pr. D. Siri, Dr. C. Lefay, Dr. D. Gigmes, Dr. Y. Guillaneuf

Aix-Marseille Université, CNRS

Institut de Chimie Radicalaire, UMR 7273

Marseille, France

E-mail:yohann.guillaneuf@univ-amu.fr

[b] J. Tran, Dr. V. Delplace, Dr. J. Nicolas

Institut Galien Paris-Sud, Univ Paris-Sud

UMR CNRS 8612, Faculté de Pharmacie

Châtenay-Malabry, France

Email: julien.nicolas@u-psud.fr

[c] Dr. I. Bataille, Dr. D. Letourneur

Laboratoire de recherche vasculaire translationnelle, INSERM 1148

University Paris 13 \& University Paris Diderot,

Paris, France

[d] Dr. C. Nicoletti, Dr. J. Perrier, Dr. M. Maresca

Aix-Marseille Université, CNRS, Centrale Marseille

UMR 7313, iSm2

Marseille, France

Supporting information for this article is given via a link at the end of the document.((Please delete this text if not appropriate)) are known to undergo beta-scission following radical addition onto the $\mathrm{C}=\mathrm{C}$ bond, thus leading to the incorporation of ester groups in the main chain through a radical mechanism. ${ }^{[16-19]}$ Copolymerization of 2-methylene-1,3-dioxepane (MDO) with propargyl acrylate, ${ }^{[13-14]}$ glycidyl methacrylate ${ }^{[11-12]}$ and 2-vinyl4,4-dimethylazlactone ${ }^{[15]}$ yielded functionalized polycaprolactone (PCL)-like copolymers that were further coupled to PEG, heparin and primary amine-bearing molecules, respectively.

Whereas this approach seems promising, since it enables the facile incorporation of various commercially available functionalized acrylate and methacrylate monomers into a PCLlike backbone, important limitations still remain. The unfavorable reactivity ratios of CKAs with traditional vinyl monomers indeed leads to considerable discrepancy between the incorporated fraction of CKA in the copolymer and its initial fraction in the monomer feed. It results in a non-statistical and thus inhomogeneous incorporation of CKA along the chain (for example, the reactivity ratio between MDO and methyl acrylate (MA) was measured ${ }^{[20]}$ as $r_{\mathrm{MDO}}=0.023$ and $r_{\mathrm{MA}}=26.53$ ). The particular reactivity of CKAs was explained by the strong nucleophilicity of the double bond. To promote radical addition onto nucleophilic olefins, electrophilic radicals are usually required. For this reason, numerous studies have been devoted to the copolymerization of CKAs with vinyl monomers leading to electrophilic radicals such as those derived from acrylate and methacrylate derivatives. ${ }^{[16,18]}$ Surprisingly, vinyl acetate (VAc), whose propagating radical is nucleophilic, yields the most favorable copolymerization results with CKAs ${ }^{[21-23]}\left(r_{\mathrm{MDO}}=0.47\right.$ and $\left.r_{\mathrm{VAc}}=1.53^{[22]}\right)$. Recently Dove and coworkers used vinyl acetate derivatives to introduce pending functionalities into $\mathrm{P}$ (MDO-co-VAc) backones. $^{[23-24]}$ The reactivity of CKA monomers is still not fully understood and a copolymerization system with CKAs that would allow for the statistical and tunable incorporation of main-chain functionalities, which would open up new and exciting perspectives in the field of degradable materials for bio-related applications, is still un unmet need.

Herein we report the discovery of the quasi-ideal copolymerization of MDO with various vinyl ether (VE) derivatives, thus enabling the facile and robust synthesis of highly functionalized polyesters via a radical mechanism (Scheme 1). Importantly, this copolymerization system takes advantage of the broad range of functional VE derivatives (some being commercially available), thus paving the way to the easy preparation of a variety of polyester-based materials with advanced properties. This method is versatile in terms of applications, as illustrated by several examples that cover functionalized polyesters, amphiphilic copolymer nanoparticles, antibacterial surfaces and cross-linked bioelastomers.

Because of the unexpected reactivity of CKAs towards vinyl monomers, we first studied the reactivity of MDO, towards (MA)- and (VAc)-based radicals using DFT calculations. The reactivity of MDO with MA and VAc was investigated as models of electrophilic and nucleophilic co-monomers, respectively. 
Frontier molecular orbitals (FMO) were first studied as they typically provide an in-depth understanding of the interactions occurring during a chemical reaction. ${ }^{\text {[25-26] }}$

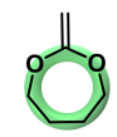

Cyclic ketene acetal (CKA)

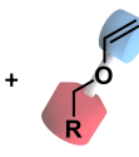

Functional vinyl ether (VE)
Free-radical copolymerization AIBN, $60-70^{\circ} \mathrm{C}$
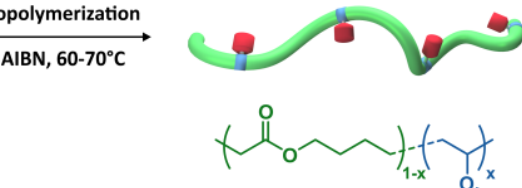

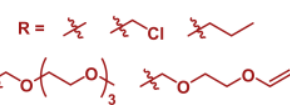

Scheme 1. Preparation of functionalized aliphatic polyester by radical ring opening copolymerization (rROP) of cyclic ketene acetal (CKA) and functionalized vinyl ether (VE) derivatives.

Monomers and radicals were calculated at the BMK/6-31G(d) level of theory since this method is efficient and provides reasonable predictions of bond dissociation energies BDEs. ${ }^{[27]}$ HOMO and LUMO orbital energies of MDO were found to be higher than those of MA, VAc and even methyl vinyl ether, which is one of the most nucleophilic monomers known, confirming the high nucleophilicity of MDO (Figure S1). FMO of radicals derived from monomers were also calculated and compared to the FMO of MDO (Figure 1). Although, as expected, 1-methoxycarbonylethyl (EEst) is more electrophilic than 2-acetyl-2-propyl (EAc), the main interaction in both cases is between the HOMO of the MDO double bond and the $\beta$-SOMO, demonstrating that both radicals behave as weak electrophilic radicals towards MDO, regardless of the philicity of the adding radical. MDO could therefore be considered as a super nucleophilic monomer when compared to traditional vinyl monomers. This could, for instance, explain the non-favored copolymerization of MDO with MA compared to its copolymerization with VAc.

Addition of various alkyl radicals onto MDO was also studied using the UB3LYP/6-31G(d) level of theory. For the calculation of activation energies, different methods and levels of theory (i.e., B3LYP, BMK and G3MP2RAD) were used since it was shown that the selected method could lead to drastic differences with experimental data (Table S1). Whatever the selected level of theory, no significant difference between the various radicals was observed, regardless of the nucleophilic or electrophilic character of the radicals. With BMK or G3(MP2)RAD, radical addition of EEst onto MDO was favored, with an activation energy $\sim 4 \mathrm{~kJ}^{\mathrm{mol}}{ }^{-1}$ lower than that of EAc. Importantly, this difference was lower than expected when considering the addition of an electrophilic radical onto a nucleophilic olefin, such as the MA and VAc model systems (see Figure S2). These calculations confirm our findings from the FMO theory: CKA monomers behave as strong nucleophilic olefins and traditional alkyl radicals are electrophilic in comparison. As a consequence, contrary to received wisdom, the difference in reactivity ratio between MA/MDO and VAc/MDO cannot be solely explained by the variation of philicity of MA- and VAc-based macroradicals as the rate of addition of EEst and EAc radicals are indeed rather similar (Table S1).
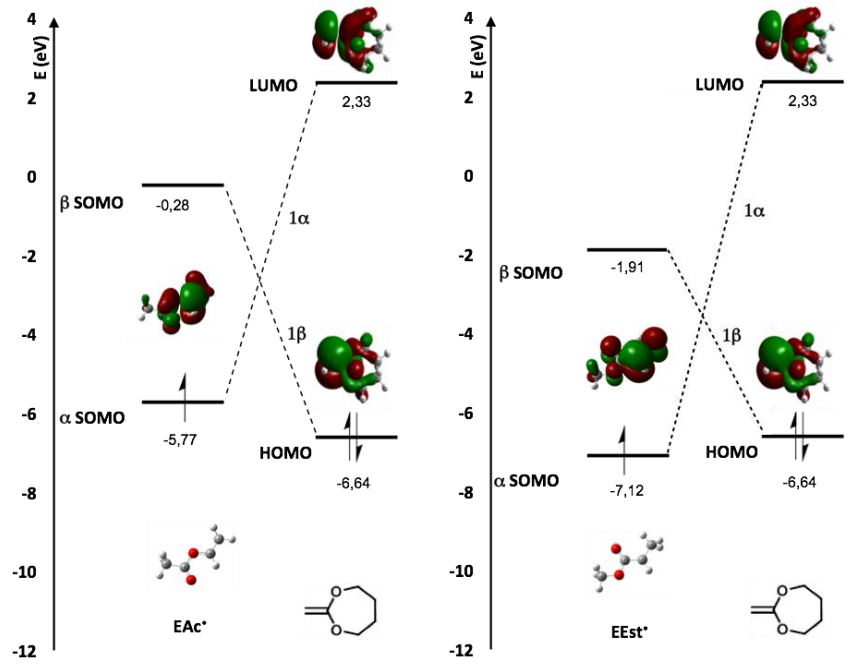

Figure 1. Frontier Molecular Orbitals (FMO) of 2-methoxycarbonyl-2-propyl (EEst) and 2-acetyl-2-propyl (EAc) radicals, and 2-methylene-1,3-dioxepane (MDO) calculated using the BMK/6-31G(d) level of theory.

The difference in reactivity observed with VAc and MA copolymerizations should instead arise from the rate of addition of vinyl-based macroradicals onto vinyl monomers $\left(k_{\text {vinyl-vinyl }}\right)$, as opposed to the rate of cross-addition of vinyl-based macroradicals onto CKA monomers $\left(k_{\text {vinyl-CKA }}\right)$. For instance, propagation rate constants of alkyl acrylates are known to be among the highest, compared to other vinyl monomers. ${ }^{[28]}$ To validate this hypothesis, all addition rate constants and reactivity ratios for MA/MDO and VAc/MDO monomer pairs were calculated and compared to the experimental values reported in the literature (Table S2). It appears that theoretical reactivity ratios of MDO with both VAc and M(M)A are in good agreement with the reported values. ${ }^{[20,22]}$

In light of these findings, we examined whether a particular vinyl monomer could lead to efficient copolymerization with CKAs. Remarkably, reactivity ratios theoretically determined for the MDO/VE pair were both close to unity, suggesting a quasiideal copolymerization behavior. To test the validity of our calculations, the free-radical bulk copolymerization of MDO and butyl vinyl ether (BVE) was investigated. The copolymerizations were performed at $70^{\circ} \mathrm{C}$ with diethyl azobisisobutyrate as radical initiator (3 mol.\%). The resulting $\mathrm{P}(\mathrm{MDO}-\mathrm{co}-\mathrm{BVE})$ copolymers were then isolated and analyzed by ${ }^{1} \mathrm{H}$ and ${ }^{13} \mathrm{C}$ NMR. ${ }^{13} \mathrm{C}$ NMR (Figure S3) confirmed the complete ring-opening of MDO. The microstructure of the copolymer was determined by 2D NMR analyses (Figure S4), revealing the presence of various diads, confirming the copolymerization reaction. Whatever the initial monomer feed ratio, the overall monomer conversions reached $\sim 70 \%$ after $15-30 \mathrm{~h}$ (Figure S5). For high initial amounts of BVE $\left(f_{\mathrm{BVE}, 0}>80 \%\right), M_{\mathrm{n}}$ were rather low $\left(\sim 4,000 \mathrm{~g} \cdot \mathrm{mol}^{-1}\right.$ with dispersity, $\Xi$, close to 1.7) because of the low reactivity of this monomer in radical polymerization. ${ }^{[29]}$ Conversely, with high initial amounts of MDO, $M_{\mathrm{n}}$ increased up to $\sim 8,000 \mathrm{~g}$. $\mathrm{mol}^{-1}$ with $\Theta$ close to 4 . Note that $\theta$ also increased due to the tendency of such polymers to undergo chain transfer reactions, leading to branching. ${ }^{[30]}$ 


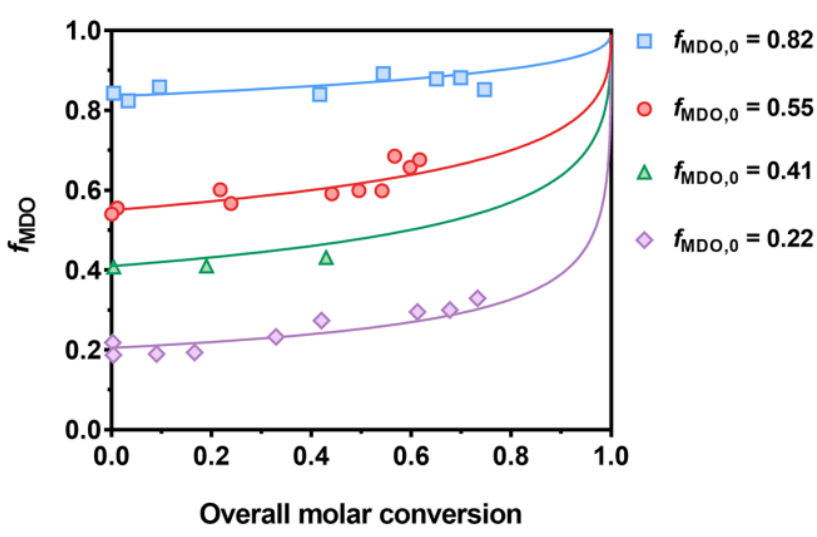

Figure 2. Experimental and theoretical MDO monomer composition versus overall molar conversion with $r_{\mathrm{BVE}}=1.61$ and $\mathrm{r}_{\mathrm{MDP}}=0.73$ during the bulk copolymerization of $\mathrm{MDO}$ and $\mathrm{BE}$ at $70^{\circ} \mathrm{C}$ initiated with $3 \mathrm{~mol} \%$ of DEAB.

Also, higher $M_{\mathrm{n}}\left(\sim 15,000 \mathrm{~g} \cdot \mathrm{mol}^{-1}, \oplus \sim 2\right)$ were obtained at $60^{\circ} \mathrm{C}$ with lower amounts of radical initiator $(0.5 \mathrm{~mol} . \%) .{ }^{[31]}$ Reactivity ratios were calculated by fitting the evolution of the feed ratio versus the overall molar conversion (Figure 2), using the Skeist equation and a non-linear least square method (NLLS) ${ }^{[32-34]}$ giving $r_{\mathrm{BVE}}=1.61$ and $r_{\mathrm{MDO}}=0.73$, in good agreement with DFT calculations. Despite a slight composition drift at high conversion, a random incorporation of the two monomers was demonstrated. This technique thus opens up new perspectives for the design of polyester-like polymer backbones with statistical incorporation of functional VE units. Indeed, since many functional VE derivatives are commercially available, facile incorporation of functional groups along the polymer backbone can be achieved. Copolymerization with ethyl vinyl ether and tetra(ethylene glycol) methyl vinyl ether were also successfully performed (Figure S6S7). It can thus be considered as an unprecedentedly robust, yet simple, route to prepare a broad range of functional polyesters for a variety of applications, as described below.

This was first illustrated by the free-radical bulk copolymerization of chloroethyl vinyl ether (CEVE) with MDO. Copolymerization between MDO and CEVE $(80: 20)$ at $70^{\circ} \mathrm{C}$ with $3 \mathrm{~mol} \%$ DEAB proceeded in $8 \mathrm{~h}$ with $\sim 40 \%$ monomer conversion for each monomer $\left(M_{\mathrm{n}}=8,800 \mathrm{~g} \cdot \mathrm{mol}^{-1}, \Xi=2.8, F_{\mathrm{MDO}}\right.$ $=0.79$ ). The $\mathrm{P}(\mathrm{CEVE}-\mathrm{co}-\mathrm{MDO})$ copolymer then served as a scaffold for post-functionalization to access to different materials (Figure 3). Pendant chlorine atoms were first efficiently functionalized with Rose Bengal through nucleophilic substitution $^{[35]}$ as observed by dual detection DRI/UV $(\lambda=350$ $\mathrm{nm})$. Pendant chlorine atoms were also quantitatively substituted by azide groups (from sodium azide, $\mathrm{NaN}_{3}$ ) for further functionalization by $\mathrm{CuAAC}^{[6-7,36]}$ (see ESI for details). This copolymer was subsequently functionalized by a small library of (macro)molecules to demonstrate the versatility of this approach: 4-pentyn-1-ol as a small model molecule and two alkyne-poly(ethylene glycol) (alkyne-PEG) of different chain lengths (750 and 2000 g.mol ${ }^{-1}$ ). 4-Pentyn-1-ol was quantitatively clicked to the copolymer as shown by the complete disappearance of the azide moieties observed by FT-IR and the quantitative appearance of all expected proton signals by ${ }^{1} \mathrm{H}$
NMR (Figure S8-S11). For alkyne-PEG derivatives, 15\% and $100 \%$ of the total amount of azide groups from P(MDO-co $\mathrm{N}_{3} \mathrm{EVE}$ ) was targeted. In both cases, CuAAC was quantitative, thus opening the door to multiple CuAAC-based postfunctionalizations. When alkyne-PEG 2000 was clicked, a shift of the SEC trace towards higher $M_{\mathrm{n}}$ was observed, accounting for successful grafting of the PEG chains (the final bimodal distribution being a result of multiple coupling between two distributions of variable shape and dispersity ${ }^{[37]}$ leading to a mixture of different polymer architectures), and the resulting material was fully soluble in water (whereas the parent copolymer was not). Interestingly, post-functionalization with the shorter PEG-chain alkyne derivative (alkyne-PEG ${ }_{750}$ ) gave selfstabilized PEGylated nanoparticles at $1 \mathrm{mg} \cdot \mathrm{mL}^{-1}\left(D_{\mathrm{z}}=151 \mathrm{~nm}\right.$, $\mathrm{PDI}=0.15)$ and $5 \mathrm{mg} \cdot \mathrm{mL}^{-1}\left(D_{z}=144 \mathrm{~nm}, \mathrm{PDI}=0.18\right)$ by nanoprecipitation (see ESI for details), thus representing a new platform for nanoparticle-based drug delivery.

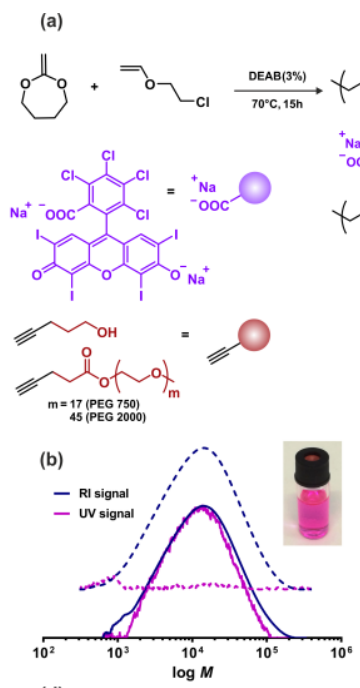

(d)

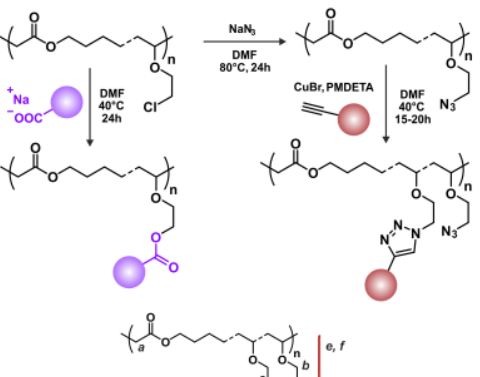

(c)
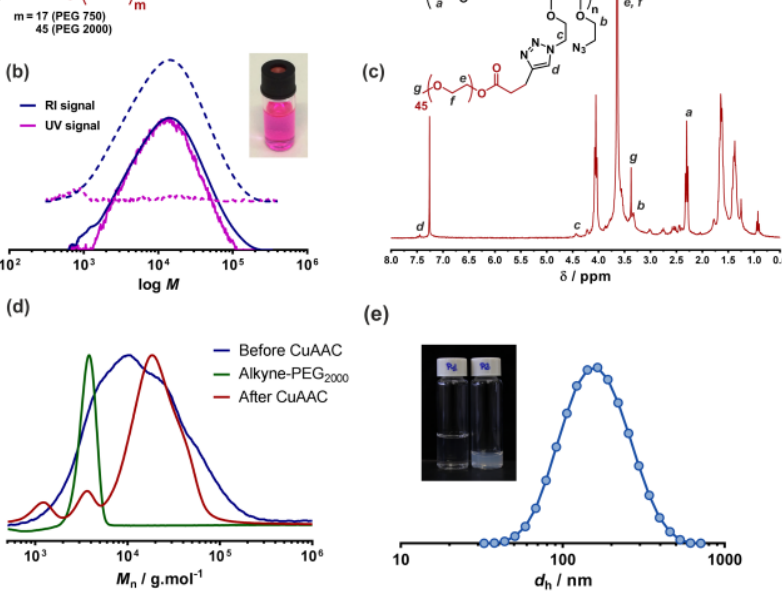

Figure 3 (a) Synthesis of poly(chloroethyl vinyl ether-co-2-methylene-1,3dioxepane) (P(CEVE-co-MDO)) copolymer and post-functionalization reactions by nucleophilic substitution by Rose Bengal or by CuAAC with pentyn-1-ol or alkyne-polyethylene glycol. (b) SEC traces (DRI/UV) of $\mathrm{P}(\mathrm{CEVE}-\mathrm{co}-\mathrm{MDO})$ copolymer before and after functionalization with Rose Bengal. Insert: picture of a solution of $\mathrm{P}(\mathrm{CEVE}-\mathrm{Co}-\mathrm{MDO})$ functionalized by Rose Bengal in THF. (c) ${ }^{1} \mathrm{H}$ NMR spectroscopy in $\mathrm{CDCl}_{3}$ of the P(CEVE-coMDO) after CuAAC with alkyne-PEG ${ }_{2000}$ (d) SEC traces (DRI) of alkyne$\mathrm{PEG}_{2000}$ (green) and $\mathrm{P}(\mathrm{CEVE}-\mathrm{co}-\mathrm{MDO})$ copolymer before (blue) and after (red) CuAAC (15\% functionalization) with alkyne-PEG ${ }_{2000}$. (e) DLS data obtained after nanoprecipitation of $\mathrm{P}(\mathrm{CEVE}-\mathrm{co}-\mathrm{MDO})$ copolymer functionalized by alkyne-PEG $750\left(5 \mathrm{mg} \cdot \mathrm{mL}^{-1}\right)$. Insert: picture of an aqueous solution of $\mathrm{P}(\mathrm{CEVE}-\mathrm{Co}-\mathrm{MDO})$ copolymer functionalized by alkyne-PEG $\mathrm{F}_{2000}$ in water ( $\mathrm{P} 1$, left) and of the nanoparticles obtained by nanoprecipitation of the P(CEVE-Co$\mathrm{MDO}$ ) copolymer functionalized by alkyne-PEG 750 at $5 \mathrm{mg} \cdot \mathrm{mL}^{-1}$ (P2, right). 
Numerous studies showed that introduction of cationic moieties onto polycarbonates and polyesters led to antimicrobial degradable materials. ${ }^{[38-40]}$ We thus tested our methodology to prepare such materials. PMDO, P(MDO-co-BVE) and P(MDOco-CEVE) films of $100 \mu \mathrm{m}$ thickness were prepared by solvent casting (Figure S12). Since PMDO is amorphous because of chain transfer processes occurring during the radical polymerization, a PCL/PMDO blend (50:50 wt.\%) was used to prepare the films, as proposed by Agarwal. ${ }^{[41]}$ This method also allowed for the simple tuning of film degradation by changing the PCL/PMDO weight ratio. The nature of the surface was probed by contact angle (CA) (Figure S13) and showed an increase of CA when butyl and chloro-ethyl functionalities were inserted. Since chlorine atoms are likely located at the film surface, the films were dipped for $30 \mathrm{~min}$ in two different amines $(\mathrm{N}, \mathrm{N}$ dimethyloctylamine $\mathrm{NMe}_{2} \mathrm{Oct}$ and triethylamine $\mathrm{Net}_{3}$ ) to anchor quaternized nitrogen at the surface (Figure 4).

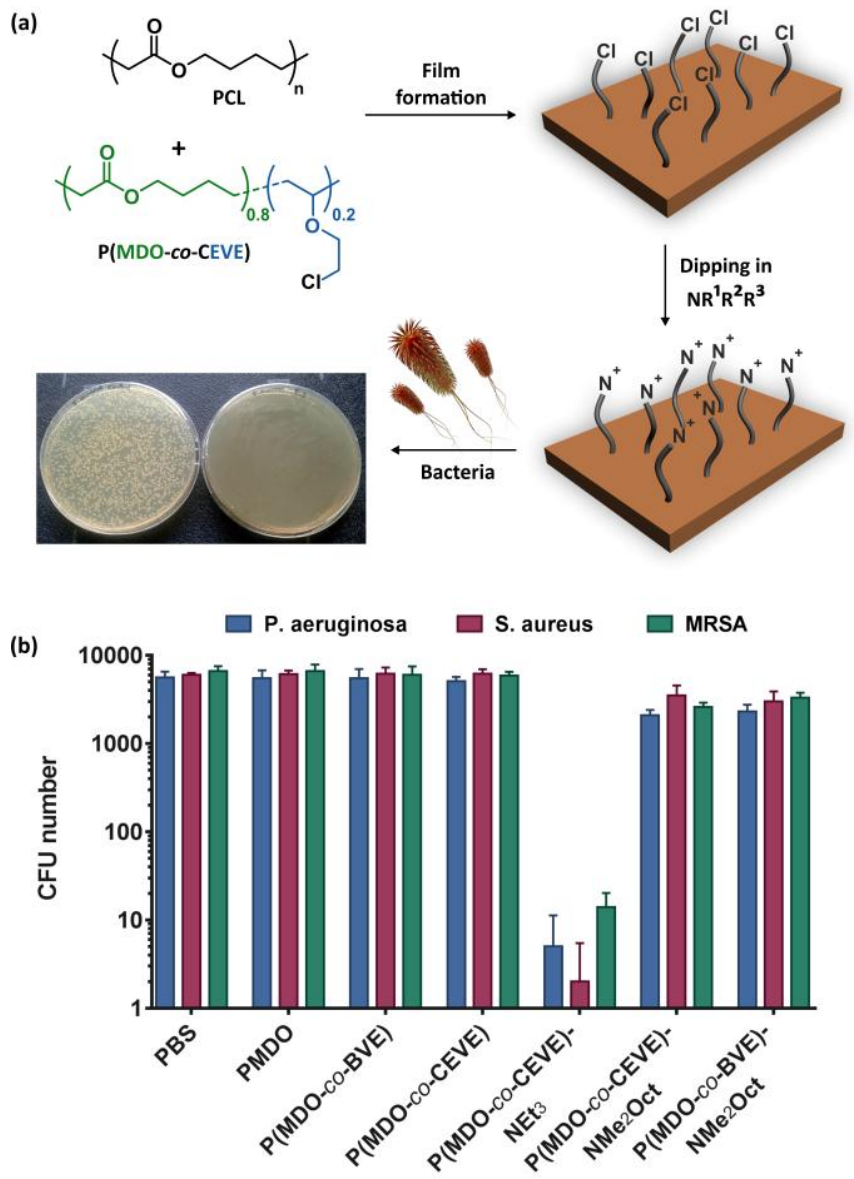

Figure 4 a) Preparation of biodegradable antibacterial surface by solvent casting from a 1:1 blend of commercially available PCL and P(MDO-co-CEVE). b) Antibacterial testing of the films on Gram- (P. aeruginosa) and Gram+ (S. aureus) bacteria, including the multiresistant strain MRSA, demonstrates that the activated film kills bacteria even after a short contact period (10 $\mathrm{min}$ ). Results are expressed as means of the number of observed viable bacteria (colony forming unit, CFU) +/- S.D with $n=3$. Insert picture is representative of the results obtained with agar plate counting showing a strong decrease in the number of viable bacteria after contact with the film (here MRSA strain). The other pictures were presented in ESI (Figure S14).
The films were further washed with water and dried at $25^{\circ} \mathrm{C}$. Their antibacterial behavior was then tested against Gram negative Pseudomonas aeruginosa, Gram positive Staphylococcus aureus and the clinical multiresistant $S$. aureus strain MRSA USA300. Films coated with quaternized triethylamine functionalities presented strong antibacterial activity, with quasi-complete bacteria killing after $10 \mathrm{~min}$ of exposure (Figure 4) as already shown by other antimicrobial degradable materials. ${ }^{[42-44]}$ Unlike triethylamine, the functionalization with $\mathrm{N}, \mathrm{N}$-dimethyloctylamine was not conclusive and did not produce a sufficiently functionalized surface to induce efficient antibacterial activity. It is important to note that control experiments (i.e., non-functionalized film dipped into $\mathrm{N}, \mathrm{N}$-dimethyloctylamine), did not exhibit any antibacterial activity, ruling out antibacterial activity from adsorbed/residual amines. Since hemolysis is the main drawbacks of cationic antibacterial polymers, the innocuity of the copolymers versus human red blood cells was also investigated (see Figure S15), and showed insignificant damage even after $4 \mathrm{~h}$ of incubation.

Beyond the use of monofunctional VEs as monomers to be copolymerized with CKAs, numerous divinyl ether derivatives are commercially available which represents an interesting opportunity for the preparation of cross-linked polyesters (often called bioelastomers ${ }^{[45-46]}$ ) via a radical mechanism. These materials are generally prepared by the polycondensation of multifunctional acid and alcohol compounds. ${ }^{[47-48]}$ Harsh conditions are usually required to prepare those materials including the final curing step. The cross-linking density and the distance between knots (i.e. covalent links between two macromolecules) being key parameters to prepare polymer networks, ${ }^{[49]}$ it is therefore essential to obtain a statistical incorporation of the cross-linker during the reaction. As the VE/CKA couple met this criteria, a copolymerization of MDO and di(ethylene glycol) divinyl ether (DIVE) (MDO:DIVE = 97:3) was investigated (Figure 5). (a)

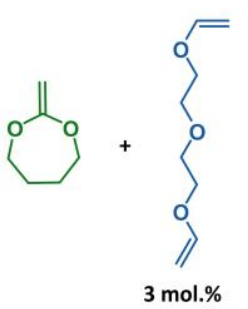

(b)

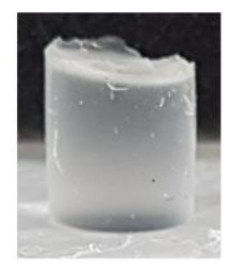

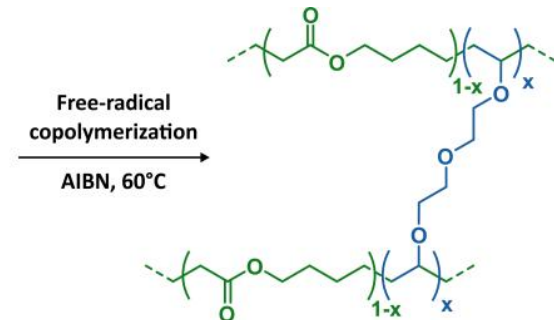

(c)

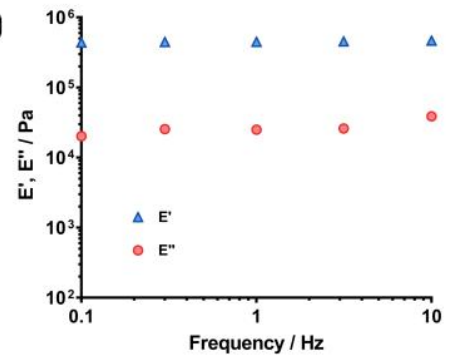

Figure 5 a) Preparation of degradable bioelastomers by the radical copolymerization of MDO and DIVE. b) Picture of the obtained material. c) Plot of storage E' and loss E" moduli as a function of frequency. 
The copolymerization was performed in prescored vials at $60^{\circ} \mathrm{C}$ with AIBN as initiator, producing the desired bioelastomer after $24 \mathrm{~h}$ of polymerization. The mechanical properties of this material were then evaluated by performing compression tests. A bioelastomer cylinder was placed in a saline solution at $37^{\circ} \mathrm{C}$ which underwent compressive DMA at $2.5 \%$ strain, with frequencies ranging from 0.1 to $10 \mathrm{~Hz}$. A complex modulus $E^{*}$ was obtained, which split up into storage E' and loss E" moduli. Both were found independent of the frequency and their respective values (450 $\mathrm{kPa}$ for E' and $25 \mathrm{kPa}$ for E") suggest typical elastic behavior (video in $\mathrm{ESI}$ ). Interestingly, the $\mathrm{E}^{\prime}$ value was comparable to the Young modulus in the linear region, and the obtained value was similar to that of poly(glycerol sebacate) PGS bioelastomers ${ }^{[48]}(280 \mathrm{kPa})$, that are widely used in tissue engineering. These results let envision a straightforward methodology for the preparation of functional PGS alternatives by copolymerizing MDO, a divinyl ether and a functional vinyl ether.

In conclusion, we demonstrated that the radical copolymerization of CKA derivatives, in particular MDO, and several commercially available vinyl ethers led to nearly random copolymers. This represents a valuable method for the preparation of functionalized polyesters with a broad range of applications.

\section{Acknowledgements}

We thank the French Ministry of Research for the financial support of the PhD thesis of A.T and the CNRS and the French National Research Agency (ANR-15-CE08-0019 and ANR-11JS08-0005) for the financial support of the PhD thesis of $\mathrm{JCH}$ and JT. This work was also supported by the 'Centre Régional de Compétences en Modélisation Moléculaire de Marseille'. Arkema is acknowledged for providing DEAB. We also thank Dr. J. Morris for proof-reading and Dr. V. Pertici for the artwork.

${ }^{\dagger}$ Those authors contributed equally to this work.

Keywords: Biomaterials • Copolymerization • Cyclic Ketene Acetals $\cdot$ DFT Calculations $•$ Functional polyesters

[1] L. S. Nair, C. T. Laurencin, Prog. Polym. Sci. 2007, 32, 762-798.

[2] Y. Ikada, H. Tsuji, Macromol. Rapid Commun. 2000, 21, 117-132.

[3] H. Seyednejad, A. H. Ghassemi, C. F. van Nostrum, T. Vermonden, W. E. Hennink, J. Control. Release 2011, 152, 168-176.

[4] R. J. Pounder, A. P. Dove, Polym. Chem. 2010, 1, 260-271.

[5] C. K. Williams, Chem. Soc. Rev. 2007, 36, 1573-1580.

[6] B. Parrish, R. B. Breitenkamp, T. Emrick, J. Am. Chem. Soc. 2005, 127, 7404-7410.

[7] R. Riva, P. Schmeits, F. Stoffelbach, C. Jerome, R. Jerome, P. Lecomte, Chem. Commun. 2005, 5334-5336.

[8] R. Riva, S. Schmeits, C. Jerome, R. Jerome, P. Lecomte, Macromolecules 2007, 40, 796-803.

[9] A. E. van der Ende, E. J. Kravitz, E. Harth, J. Am. Chem. Soc. 2008, 130, 8706-8713.

[10] B. Parrish, T. Emrick, Bioconjugate Chem. 2007, 18, 263-267.
[11] J. Undin, A. Finne-Wistrand, A.-C. Albertsson, Biomacromolecules 2014, 15, 2800-2807.

[12] J. Undin, A. Finne-Wistrand, A.-C. Albertsson, Biomacromolecules 2013, 14, 2095-2102.

[13] S. Maji, F. Mitschang, L. Chen, Q. Jin, Y. Wang, S. Agarwal, Macromol. Chem. Phys. 2012, 213, 1643-1654.

[14] S. Maji, M. Zheng, S. Agarwal, Macromol. Chem. Phys. 2011, 212, 2573-2582.

[15] M. C. D. Carter, J. Jennings, V. Appadoo, D. M. Lynn, Macromolecules 2016, 49, 5514-5526.

[16] S. Agarwal, Polym. Chem. 2010, 1, 953-964.

[17] W. J. Bailey, Z. Ni, S.-R. Wu, J. Polym. Sci. Polym. Chem. 1982, 20 , 3021-3030.

[18] A. Tardy, J. Nicolas, D. Gigmes, C. Lefay, Y. Guillaneuf, Chem. Rev. 2017, 117, 1319-1406.

[19] V. Delplace, J. Nicolas, Nat. Chem. 2015, 7, 771-784.

[20] L. F. Sun, R. X. Zhou, Z. L. Liu, J. Polym. Sci.: Part A: Pol. Chem. 2003 41, 2898-2904.

[21] J. Undin, T. Illanes, A. Finne-Wistrand, A.-C. Albertsson, Polym. Chem. 2012, 3, 1260-1266.

[22] S. Agarwal, R. Kumar, T. Kissel, R. Reul, Polym. J. 2009, 41, 650-660.

[23] G. G. Hedir, C. A. Bell, R. K. O'Reilly, A. P. Dove, Biomacromolecules 2015, 16, 2049-2058.

[24] G. G. Hedir, C. A. Bell, N. S. leong, E. Chapman, I. R. Collins, R. K. O'Reilly, A. P. Dove, Macromolecules 2014, 47, 2847-2852.

[25] Y. Li, J. N. S. Evans, J. Am. Chem. Soc. 1995, 117, 7756-7759.

[26] B. Giese, Angew. Chem. Int. Ed. 1983, 22, 753-764.

[27] B. B. Noble, M. L. Coote, Int. Rev. Phys. Chem. 2013, 32, 467-513.

[28] J. M. Asua, S. Beuermann, M. Buback, P. Castignolles, B. Charleux, R. G. Gilbert, R. A. Hutchinson, J. R. Leiza, A. N. Nikitin, J. P. Vairon, A. M. van Herk, Macromol. Chem. Phys. 2004, 205, 2151-2160.

[29] A. Matsumoto, T. Nakana, M. Oiwa, Makromol. Chern., Rapid Commun 1983, 4, 277-279.

[30] S. Jin, K. E. Gonsalves, Macromolecules 1997, 30, 3104-3106.

[31] A. Tardy, J.-C. Honoré, D. Gigmes, J. Nicolas, C. Lefay, Y. Guillaneuf, Polym. Chem. 2017, DOI: 10.1039/c1037py00337d.

[32] A. M. Vanherk, J. Chem. Educ. 1995, 72, 138-140.

[33] A. M. vanHerk, T. Droge, Macromol. Theory Simul. 1997, 6, 1263-1276.

[34] L. Couvreur, B. Charleux, O. Guerret, S. Magnet, Macromol. Chem. Phys. 2003, 204, 2055-2063.

[35] L. Pessoni, S. Lacombe, L. Billon, R. Brown, M. Save, Langmuir 2013, 29, 10264-10271.

[36] N. S. Teske, J. Voigt, V. P. Shastri, J. Am. Chem. Soc. 2014, 136, 10527-10533.

[37] C. Barner-Kowollik, Macromol Rapid Commun 2009, 30, 1625-1631.

[38] R. Riva, P. Lussis, S. Lenoir, C. Jerome, R. Jerome, P. Lecomte, Polymer 2008, 49, 2023-2028.

[39] F. Nederberg, Y. Zhang, J. P. K. Tan, K. J. Xu, H. Y. Wang, C. Yang, S. J. Gao, X. D. Guo, K. Fukushima, L. J. Li, J. L. Hedrick, Y. Y. Yang, Nat. Chem. 2011, 3, 409-414.

[40] A. C. Engler, N. Wiradharma, Z. Y. Ong, D. J. Coady, J. L. Hedrick, Y. Y. Yang, Nano Today 2012, 7, 201-222.

[41] S. Agarwal, C. Speyerer, Polymer 2010, 51, 1024-1032.

[42] L. Tan, S. Maji, C. Mattheis, Y. Chen, S. Agarwal, Macromol. Biosci. 2012, 12, 1721-1730.

[43] H. Wang, C. V. Synatschke, A. Raup, V. Jerome, R. Freitag, S. Agarwal, Polym. Chem. 2014, 5, 2453-2460.

[44] Y. Sun, G. Sun, J. Applied Polym. Sci. 2001, 80, 2460-2467.

[45] C. J. Bettinger, Macromol Biosci 2011, 11, 467-482.

[46] Q. Chen, S. Liang, G. A. Thouas, Prog. Polym. Sci. 2013, 38, 584-671.

[47] J. Yang, A. R. Webb, G. A. Ameer, Adv. Mater. 2004, 16, 511-516.

[48] Y. Wang, G. A. Ameer, B. J. Sheppard, R. Langer, Nat. Biotechnol. 2002, 20.

[49] Q. Liu, M. Tian, R. Shi, L. Zhang, D. Chen, W. Tian, J. Applied Polym. Sci. 2007, 104, 1131-1137. 


\section{Entry for the Table of Contents}

\section{COMMUNICATION}

VE/CKA: the ideal couple. The radical copolymerization between cyclic ketene acetal monomers and vinyl ether derivatives led to a new and easy way to prepare homogeneously functionalized aliphatic polyesters (nanoparticles, antibacterial surfaces and bioelastomers).

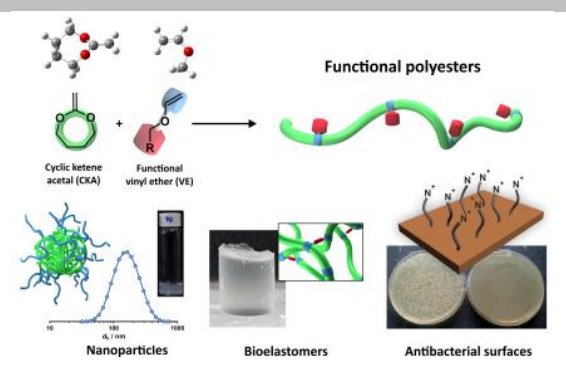

Antoine Tardy, Jean-Claude Honoré, Johanna Tran, Didier Siri, Vianney Delplace, Isabelle Bataille, Didier Letourneur, Josette Perrier, Cendrine Nicoletti, Marc Maresca, Catherine Lefay, Didier Gigmes, Julien Nicolas, Yohann Guillaneuf*

Page No. - Page No.

Radical Copolymerization of Vinyl Ethers and Cyclic Ketene Acetals as a Versatile Platform to Design Functional Polyesters 